\title{
El diseño curricular y la inclusión de la dimensión ambiental en una carrera universitaria
}

\author{
Licda. María Carlota Avila Arbizú de Palacios \\ lotyavila@gmail.com \\ Universidad de San Carlos de Guatemala \\ Centro Universitario de Sur Oriente CUNSURORI-Jalapa \\ Mtra. Yadira Abigail Ishlaj Conde de González \\ yadirabic@hotmail.com \\ Universidad de San Carlos de Guatemala \\ Centro Universitario de Sur Oriente CUNSURORI-Jalapa
}

Fecha de recepción: 15/11/2015 Fecha de aceptación: 05/04/2016

\begin{abstract}
Resumen
Los diseños curriculares dentro de las instituciones educativas son indispensables, porque constituyen un documento que proyecta, fundamenta y dirige la ejecución de las actividades académicas, laborales e investigativas, por lo que sirven de orientadores de modelos metodológicos en donde se permite la participación de los estudiantes y se realizan procesos de extensión comunitaria. Contempla la formación de los estudiantes en función de un proceso de enseñanza integral relacionado con el contexto en donde habrán de insertarse los egresados en el mercado laboral. La estructuración de un diseño curricular implica la expresión clara y precisa de cada uno de los aspectos vinculados a: pensa de estudios, contenidos, áreas y ejes de formación, procesos de enseñanza y aprendizaje y el establecimiento de normas básicas de especificación sobre: procesos de evaluación y mejoramiento de los contenidos y procesos de enseñanza y aprendizaje. Se constituye en un instrumento común en la interacción de los profesionales que conforman el equipo docente. Los diseños curriculares en la Universidad de San Carlos de Guatemala, se encuentran estructurados de acuerdo a las disposiciones legales, administrativas, académicas y de desarrollo curricular; elementos que se consideran en el desarrollo del diseño curricular de la Carrera de Pedagogía a nivel de pre-grado.
\end{abstract}

\section{Palabras Clave}

Diseño curricular, currículo, organización curricular, ambientalización curricular, carrera de pedagogía.

\begin{abstract}
Curricular designs inside educative institutions are indispensable, they constitute documents that project, fundament and direct execution of activities in research, academic and labor fields. So, they serve as orienteer of methodological processes where participation of students are allowed and extension processes are made within the community. These designs often contemplate students formation within a process of integral teaching related on a context where graduates should integrate labor market. Structure of a curricular design implies clear and precise expression of each and every aspect related to: Studies Program, contents, areas and formation axles, teaching and learning procedures and setting of basic rules about: Assessment processes, content improvement and teaching and learning processes. They are common instruments in teaching team interaction. Inside our University, Curricular designs are structured according to legal, academic, administrative and curricular development dispositions; all these elements are considered in order to develop a curricular design on Education and Pedagogics degree on a technical level.
\end{abstract}

\section{Key Words}

Curricular design, curriculum, curricular organization, pedagogics degree. 


\section{Introducción}

La apertura e inicio de funciones en el año 2009 de la Carrera de Profesorado de Enseñanza Media en Pedagogía y Técnico en Administración del Centro Universitario de Sur Oriente CUNSURORI-Jalapa, amplió la oferta educativa a plan fin de semana para la población universitaria, con lo que la matrícula estudiantil del centro universitario aumentó considerablemente de forma escalonada conforme se apertura los semestres. A la fecha se han abierto dos sedes más en los municipios de Mataquescuintla y San Pedro Pinula y se trabaja en cuatro secciones, tres plan sábado y una plan domingo.

En cuanto al desarrollo curricular el pensum de estudios con el cual se trabaja corresponde al que se implementa en la Facultad de Humanidades del campus central y al que se utilizó en la sección de Jutiapa. Sin embargo, no se cuenta con un documento escrito en donde se concrete el diseño curricular de la carrera y que exprese la intencionalidad en la formación del egresado, lo que conlleva a reflexionar en torno al proyecto curricular con el cual se trabaja para la formación del estudiante y su vinculación con el contexto nacional en materia educativa y entre los perfiles de egreso del Profesor de Enseñanza Media que se forma actualmente en el CUNSURORI.

Se debe considerar que la formación del estudiante de pedagogía requiere de un diseño curricular acorde a los requerimientos del contexto social y del mercado laboral, pues actualmente se demanda profesionales competentes que afronten la carrera docente con las herramientas adecuadas para el abordaje de los paradigmas pedagógicos que se implementan en el Sistema Educativo Nacional.

En virtud de lo cual se demanda que el profesional posea criterio y liderazgo que le permitan ser actor decisorio e innovador en los procesos educativos de acuerdo a la implementación del diseño curricular implantado a nivel nacional a través del Currículo Nacional Base, CNB. Por tanto, el perfil de egreso debe encaminarse a implantar en el estudiante las competencias necesarias que le posibiliten para enfrentar el mercado laboral con los insumos adecuados para operativizar los diseños curriculares con los cuales trabaja el Ministerio de Educación, MINEDUC, en el ciclo de educación media.

En función de ello se implementó el estudio de investigación "Propuesta de Diseño Curricular para la Carrera de Profesorado de Enseñanza Media en Pedagogía y Técnico en Administración Educativa del Centro Universitario de Sur Oriente, CUNSURORI-Jalapa", en donde se realizó las indagaciones en tres líneas de dirección: Coordinación de Carrera, Docentes y Estudiantes, para obtener una visión integral de la situación y establecer generalidades para su análisis.

Los resultados obtenidos permitieron la construcción del diagnóstico curricular de la carrera y el análisis respecto a la inclusión del componente ambiental, que se concreta en la Propuesta de Diseño Curricular de la Carrera de Profesorado de Enseñanza Media en Pedagogía y Técnico en Administración Educativa del CUNSURORI-Jalapa, como proyecto orientador del ejercicio académico en las sedes en donde se imparte la carrera.

La construcción de la estructura curricular que se diseñó se vincula con los elementos legales, académicos y filosóficos de la universidad; además se incorpora lo contenido en el Plan Estratégico USAC-2022 que en materia curricular y ambiental fundamentan la propuesta. Por ser la única carrera que dentro del CUNSURORI trabaja con la formación de formadores se necesita establecer con claridad los componentes estructurales del currículo y la determinación de áreas y ejes de formación vinculantes a las disposiciones emanadas por la universidad.

La incorporación de la dimensión ambiental dentro del diseño curricular se aborda como una concepción integradora de conocimientos, hábitos, habilidades, actitudes y valores, conscientemente diseñado y contextualizado, que 
atraviese todo el plan de estudios, de manera que quede establecido qué aportó cada uno de los contenidos en el proceso docente educativo y que dé como resultado una formación integral en el sujeto, que se exprese en su actuación hacia el medio, respecto a su entorno y a la problemática ambiental (Covas, s.f.).

\section{Diseño curricular}

El diseño curricular se concibe como un documento normativo que sirve como medio para hacer operativo el currículo. Articula las orientaciones filosóficas, epistemológicas, sociológicas, antropológicas, psicopedagógicas y andragógicas para imprimir direccionalidad al proceso educativo (Secretaría de Estado de Educación y Cultura, 2000), por las caraterísticas de la población a atender dentro de la carrera y el rango de edades (se estima que se cuenta con estudiantes de 17 a 55 años) que se matriculan anualmente, se incorporan además las orientaciones andragógicas que sustentan el diseño curricular. Su implantación dentro de la institución direcciona la planeación educativa y se sustenta de la teoría curricular, para lo cual se consideran sus fundamentos:

\subsection{Fundamento legal}

Se refiere al espíritu de las principales normas que sustentan el currículo, el marco legal orienta y regula la educación. En el caso de la universidad la Constitución Política de la República de Guatemala y la Ley Orgánica de la Universidad regulan lo relacionado al proceso de diseño curricular.

\subsection{Fundamento filosófico}

El currículo se estructura en beneficio de los individuos y de la sociedad, y debido a esta responsabilidad, el mismo está permeado de visiones filosóficas. El enfoque filosófico que sustenta el currículo en las unidades formadoras, obedece a la línea de pensamiento que la institución determina incorporar en el diseño curricular.
Se debe considerar que el ser humano está condicionado por las relaciones sociales existentes en su entorno y por las exigencias, aspiraciones y características de la civilización universal (interdependencia).

Los aspectos filosóficos en el currículo concentran una carga epistemológica, ontológica y axiológica, categorías que cobran relevancia en la fundamentación a nivel curricular en tanto conducen a responder ¿Qué debe la persona ser?, ¿Qué puede lograr ser? y ¿Qué podemos enfatizar?, la respuesta que se dé a estas interrogantes direccionará las preceptos generales de los componentes curriculares.

\subsection{Fundamento sociológico}

Hace referencia al conjunto de demandas que la sociedad realiza a un proyecto de formación determinado y tiene la finalidad de adecuar ese proyecto a las necesidades, valores y expectativas sociales (Delgado, 2013).

La sociedad tiene mecanismos para facilitar el cambio, tales como las estructuras legales y legislativas. Existen ciertas áreas en la sociedad donde el ajuste se hace más difícil. Entre estas están la moral, la religión, los aspectos sexuales, y otras. La educación ha ayudado a que se reconozcan y mantengan en forma continua ciertos patrones. Entre éstos la formulación de objetivos que enfatizan mayormente la dimensión cognoscitiva del aprendizaje, la organización de la experiencia educativa por grados, el énfasis en la organización tradicional del contenido en asignaturas, la evaluación basada principalmente en el aprovechamiento.

\subsection{Fundamento epistemológico}

Se refiere a las exigencias que imponen al diseño curricular las características propias de las disciplinas que se toman como referencia al seleccionar y organizar los contenidos (Delgado, 2013), esta fuente es la que se origina de las disciplinas científicas y contribuye a la búsqueda de su estructura interna, su constructo y su concepción (Coll, 1987). 
Los fundamentos epistemológicos dan cuenta de las condiciones a través de las cuales se produce el conocimiento, se define los enfoques, paradigmas y posibilita la presencia de la pedagogía, la didáctica y el currículo para la objetivación de la enseñanza, a través del diseño y desarrollo de estrategias de enseñanza y de aprendizaje.

\subsection{Fundamento psicológico}

Considera las concepciones y teorías sobre el desarrollo y el aprendizaje que se toman como referencia a la hora de decidir la metodología, la selección y formulación de los objetivos y organización de contenidos (Delgado, 2013).

Además de considerar los niveles de desarroIlo intelectual, el educador debe considerar el desarrollo total de los individuos, pues estos junto con los cambios sociales le proveen al educador ideas sobre el propósito general del currículo.

\subsection{Fundamento pedagógico}

Tiene que ver con las concepciones teóricas que uno sostiene de cómo se debe enseñar, tanto en general, como en lo que afecta a una determinada materia o contenido (Delgado, 2013).

Los procesos enseñanza y aprendizaje se administran según las diferencias individuales, sociales y regionales del país con marcos conceptuales, actitudes y destrezas del estudiante, que le permitan ajustarse, integrarse o transformar la realidad físico-cultural en la que habrá de intervenir.

Al respecto Beauchamp (1977) citado por Aguilar Morales et. al., refiere que el diseño curricular forma parte esencial de la planeación educativa. El término currículo puede tener tres acepciones: a) un documento que será el punto de partida para planear la instrucción, b) un sistema de planeación, y c) un campo de estudio que abarca el diseño curricular, la ingeniería curricular y la investigación-teoría necesaria para fundamentar el diseño y la ingeniería curricular.

El diseño curricular puede entenderse como una dimensión del currículum que revela la metodología, las acciones y el resultado del diagnóstico, modelación, estructuración y organización de los proyectos curriculares. Prescribe una concepción educativa determinada que al ejecutarse pretende solucionar problemas y satisfacer necesidades y en su evaluación posibilita el perfeccionamiento del proceso enseñanza-aprendizaje (Arnaz, 1996).

Su implementación dentro de las instituciones educativas permite orientar el proceso formativo de sus estudiantes en función de la formación del perfil de egreso del profesional que desea titularse. Para lo cual se debe considerar la estructuración del diseño curricular acorde a los lineamientos establecidos.

La universidad cuenta con una transformación curricular efectiva y permanente que hace posible la flexibilidad en el diseño y rediseño curricular, que le permite tener amplia y diversa oferta académica, acorde con la acreditación de la formación universitaria a nivel regional. Participa activamente a nivel nacional en los procesos de Reforma Educativa (Universidad de San Carlos de Guatemala, USAC, 2003:11).

\subsection{Fundamento andragógico}

La andragogía se ha fundamentado y trabajado en tres grandes campos: como disciplina científica, como proceso educativo integral y como actividad profesional. Como disciplina científica, la andragogía ha desarrollado un cospus teórico, fundamentado en principios filosóficos, psicológicos y sociológicos que le otorgan características propias y distintivas al diseño e implementación de procesos educativos para las personas adultas; así como un conjunto de conocimientos sobre su historia, metodología y propuestas. 
Se considera que el hecho andragógico incorpora dos actores principales: el participante y el facilitador(a) y se basa en dos principios fundamentales, la horizontalidad y la participación, por lo que se distingue como un proceso real, objetivo y concreto en cuanto: existe el sujeto adulto como una realidad biológica, económica y social, el adulto es susceptible de educarse en todas las etapas de su vida y la sociedad necesita educar a sus miembros. Precisa el diseño de estrategias de enseñanza y aprendizaje apropiadas a la etapa adulta y que se enmarquen para su implementación bajo las condiciones de: confrontación de experiencias, racionalidad, capacidad de abstracción del adulto e integración y aplicabilidad.

De tal forma que la atención que se proporcione al estudiante en la educación superior responda a las condiciones y motivaciones propias del adulto, en donde el andragogo diseñe estrategias que sitúen al participante en el proceso de orientación-aprendizaje y sea estimulado a un pensamiento analítico, crítico y creativo para la transformación y mejoramiento de su entorno.

\section{Currículo}

Existe variedad de puntos de vista respecto a la construcción de una definición de currículo consensuada, según la postura de diversos autores su perspectiva y fundamentación teórico-metodológica plantean diversas acepciones. Al respecto Mauri señala que:

$\checkmark \quad$ Para quienes consideran que lo importante es una buena planificación de la enseñanza, el currículum debe ser un plan de acción.

$\checkmark$ Si lo que realmente importa es lo que ocurre con el alumno en cuanto aprendizaje de conocimientos, modificación de actitudes y desarrollo de valores, tiende a definirse el currículum como las experiencias que, planificadas o no, tienen lugar en la escuela. $\checkmark \quad$ Si ponen el énfasis en los factores introducidos por el profesor, considerando como el verdadero modelador de las situaciones de enseñanza-aprendizaje, se inclinan por concebirlo como el arte, difícilmente planificable, de abordar los problemas que se plantean con los individuos en las situaciones de enseñanza-aprendizaje.

$\checkmark$ Quienes centran su atención en aquello que debe ser enseñado, se orientan hacia una concepción del currículum como conjunto y los principios de selección y organización de los contenidos objetos de enseñanza y de aprendizaje (Mauri, 1990).

El currículo expresa la intencionalidad de la educación, orienta y organiza la práctica educativa a la vez que es confrontado constantemente con la realidad en la que se aplica. Su naturaleza refiere que debe estar sujeto a un proceso de revisión y ajuste que permita secuenciar mejor los contenidos contemplados en los planes de estudios y los objetivos orientadores en la formación del estudiante, todo esto en función de los requerimientos del contexto social y el mercado laboral.

La Universidad de San Carlos de Guatemala, como miembro del Consejo Superior Universitario Centroamericano -CSUCA- , y única institución de educación superior estatal en Guatemala, desarrolla su estructura curricular según los mandatos constitucionales de responder a las necesidades de formar el recurso humano profesional universitario, que se requiere para contribuir al desarrollo permanente de la nación. (González de Ochaeta, 2005:21)

El SICEVAES ${ }^{1}$ es el sistema que promueve la cultura de calidad, la evaluación y el rendimiento de cuentas a la sociedad en las universida-

1 Sistema Centroamericano de Evaluación y Acreditación de la Educación Superior (SICEVAES), promueve la cultura de calidad, evaluación y el rendimiento de cuentas a la sociedad en las universidades adscritas, utiliza la evaluación y acreditación como estrategia de gestión del cambio, actualización, modernización y mejoramiento de las universidades. 
des miembros, usando la evaluación y acreditación como estrategia de gestión del cambio, modernización y mejoramiento de las universidades. Asimismo, promueve el establecimiento de mecanismos regionales de acreditación internacional de la calidad de la educación universitaria de América Central. Además, el SICEVAES promueve la armonización e integración académica regional de la educación superior centroamericana. (CSUCA, 2013:19).
La construcción del currículo de estudios de la Carrera de Pedagogía debe enmarcarse dentro de las disposiciones generales que emanan de la USAC, el diseño curricular debe fundamentarse en los marcos legales y filosóficos de la institución educativa y no meramente una construcción propia de la carrera, en donde la concreción curricular debe ser asumida por diversos actores en el proceso a la vez que los vincula de forma progresiva en su implantación. M sistema.

\section{Esquema 1}

\section{Niveles de Concreción Curricular}

Agente responsable

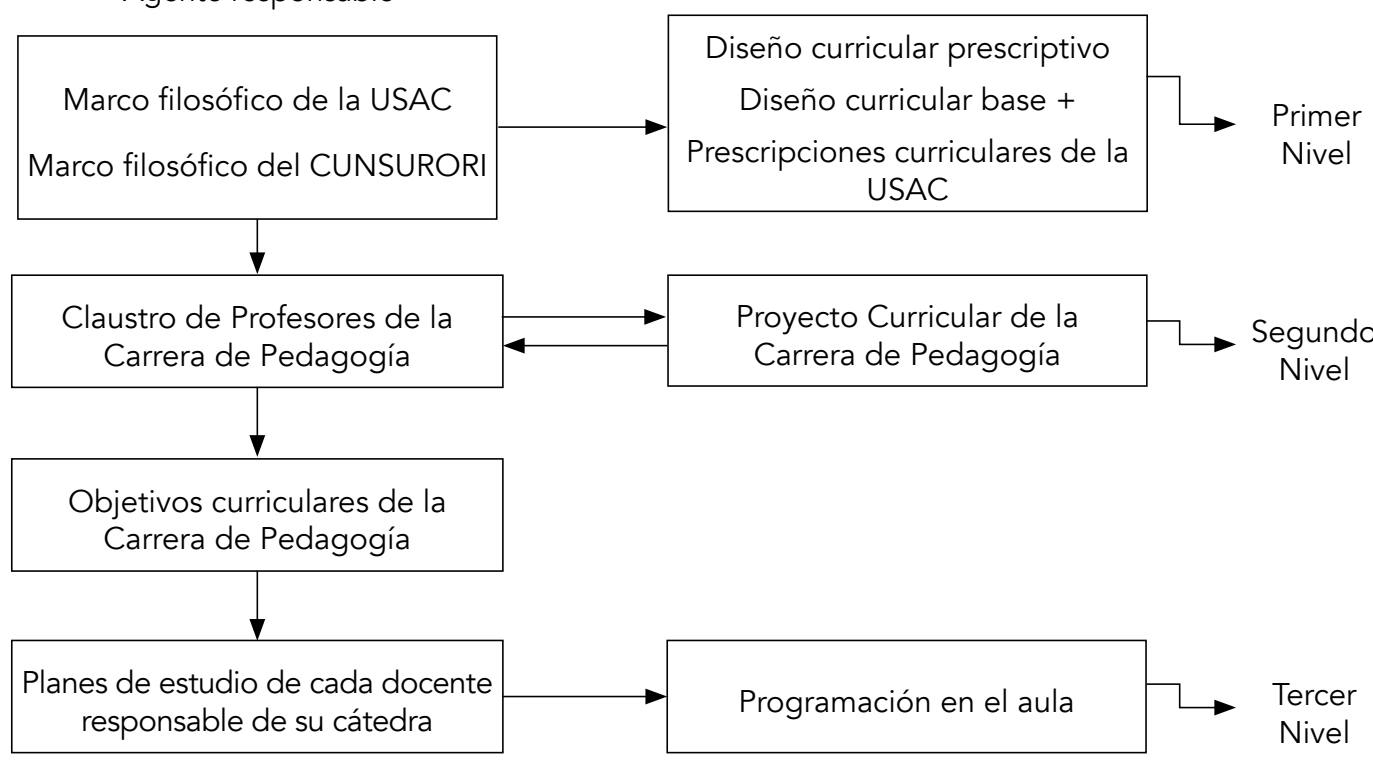

Fuente: Elaboración propia, tomando como referencia los niveles de concreción curricular planteados por Ander Egg, 1990.

Llevar a cabo una planeación curricular significa, además de tomar en cuenta los propósitos para los que fue creado, estar abierto a su reestructuración y que sea posible su realización; debe tomar en cuenta también el momento histórico en que se desarrolla y la cultura a la que se pretende hacerlo llegar (Meza, 2012:9).

\section{Clasificación del currículo}

Existen diversas clasificaciones en torno a los tipos de currículo que se implementan en las instituciones educativas. En su obra Análisis del Currículum Posner (1998) nos presenta una tabla contentiva de lo que el autor denominaba los cinco currícula simultáneos, ellos son:

\subsection{Currículo oficial}

Descrito en forma documental, a través de planes y programas, materiales didácticos sugeridos, guías curriculares y los objetivos que el sistema educativo vigente aspire alcanzar mediante la aplicación de esos planes. La experiencia nos ha demostrado que el currícu- 
lum oficial no es inflexible, ya que en la puesta en práctica de lo planificado intervienen diversos elementos humanos, materiales y circunstanciales que lo hacen dinámico y, por tanto, sujeto a modificaciones valederas.

\subsection{Currículo operacional}

Currículum operacional: incorporado en las prácticas y pruebas de enseñanza, también se le denomina currículum pertinente, concebido como el resultado de la aplicabilidad y utilidad del currículum, cuando se pasa de la teoría (como estudiantes universitarios) a la práctica (en el desenvolvimiento como profesionales).

\subsection{Currículo oculto}

Currículum oculto: representado por las normas institucionales y valores no reconocidos abiertamente por profesionales y funcionarios escolares, su profundidad e impacto a veces llega a tener resultados mayores que los del currículum oficial.

\subsection{Currículo nulo}

Currículum nulo: tema de estudio no enseñado, que al formar parte del currículum no tiene aplicabilidad ni utilidad aparente, llega a considerarse como materias y contenidos superfluos.

\subsection{Extra currículum}

Son las experiencias planeadas, externas al currículum oficial, es de carácter voluntario y está vinculado con los intereses estudiantiles.

Así también se encuentra la distinción que realiza Rohlehr, es importante establecer la distinción entre el currículo previsto, escrito, enseñado y aprendido. El currículo previsto representa las directrices formales aprobadas que se vierten a un currículo escrito que incluye todo lo necesario para su implementación. Posteriormente el currículo escrito se transforma en el currículo enseñado a través del impacto de los resultados de aprendizaje, la aplicación de métodos pedagógicos eficaces y el monitoreo de su efectividad en el aula.

El aprendizaje debe construirse sobre la base de experiencias y logros previos y preparar al estudiante para continuar aprendiendo. Esta es la esencia del concepto continuidad en el currículo (Rohlehr, 2006). 


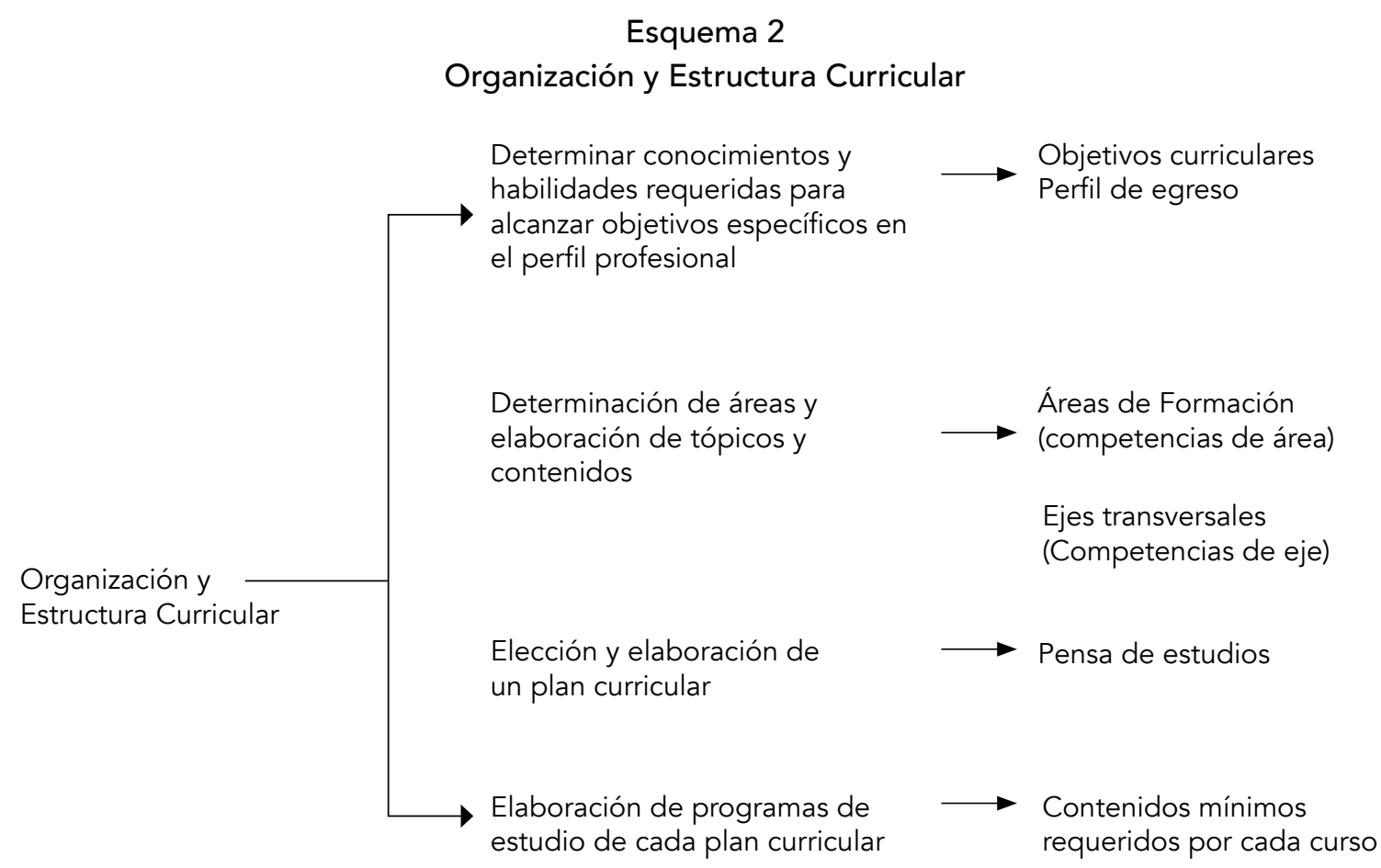

Fuente: Elaboración propia, agosto 2014.

La organización y estructura curricular permite determinar los componentes que se consideran en el diseño curricular de la carrera, aspectos que se perfilan de acuerdo a las características propias del contexto social en que habrá de desempeñarse el egresado.

\section{Organización de la estructura curricular}

Esta tarea consiste en la secuenciación y estructuración de los componentes que intervienen en el proyecto curricular que se diseña. En este momento se determina el orden en que se va a desarrollar, el lugar de cada componente, el tiempo necesario, las relaciones de precedencia e integración horizontal necesarias y todo ello se lleva a un mapa curricular, donde quedan reflejadas todas estas relaciones. El aprendizaje debe constituirse sobre la base de experiencias y logros previos y preparar al estudiante para continuar aprendiendo. Esta es la esencia del concepto de continuidad en el currículo (Rohlehr, 2006).
La organización y estructura curricular permite determinar los conocimientos y habilidades requeridas para alcanzar objetivos específicos del perfil profesional, esto a través de la determinación de áreas y elaboración de tópicos y contenidos que permitan la construcción de un plan curricular y la elaboración de programas de estudio para cada plan curricular. Los componentes curriculares se encuentran conformados por: objetivos curriculares, áreas de formación, ejes curriculares, ejes transversales, perfil de egreso y programas básicos o de cursos.

\subsection{Objetivos}

La formulación de los objetivos curriculares, es decir definir lo que se ha de obtener en el término del proceso educativo, implica en primer lugar, la precisión de las necesidades de aprendizaje, en seguida caracterizar al alumno insumo, a continuación elaborar el perfil de egresado y por último definir propiamente los objetivos curriculares. (Aguilar \& Vargas, 2011:56). 


\section{Esquema 3}

Formulación de los Objetivos Curriculares

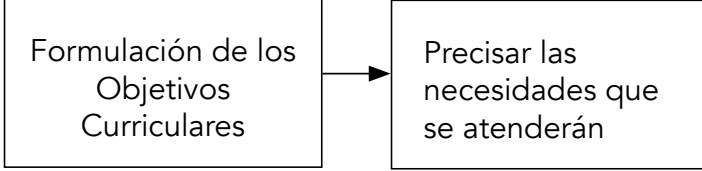

Fuente: Arnaz, 1990:18

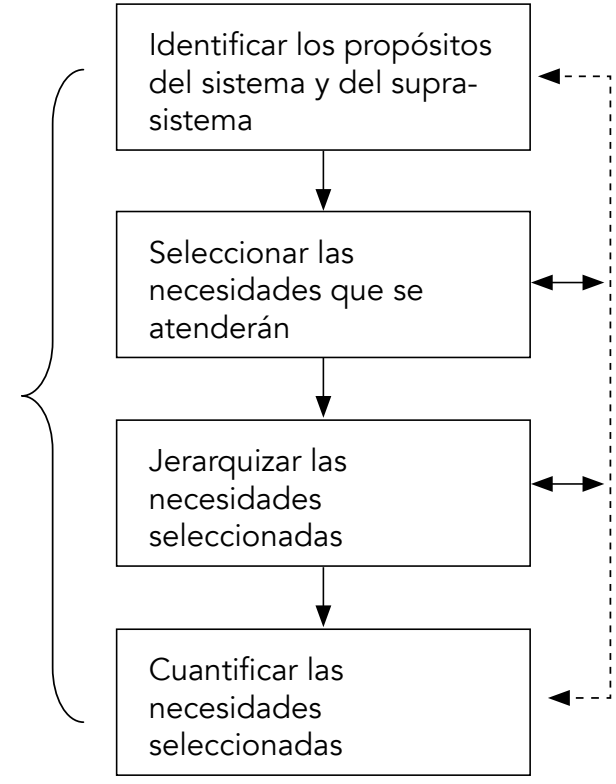

Identificar los propósitos del sistema y del suprasistema

Seleccionar las necesidades que se atenderán
Formular los objetivos curriculares es una operación que ha de iniciarse con la actividad destinada a precisar las necesidades que se atenderán (Arnaz, 1990:18), a través de las cuales se determine los propósitos orientadores del currículo bajo las directrices que al respecto norman la estructuración del diseño curricular en la USAC, lineamientos generales que emanan de la Dirección General de Docencia, DIGED, la cual tiene como finalidad velar por el mejoramiento del sistema educativo a través de la investigación educativa, asesoría y desarrollo curricular, evaluación y formación de profesores (González de Ochaeta, 2005). La estructuración de los objetivos curriculares permite la identificación de las áreas de formación que integrarán el currículo.

\section{2 Áreas de formación}

Se considera como áreas de formación al conjunto de materias agrupadas por áreas de estudio, en orden al perfil de egreso y distribui- das en el tiempo o duración del currículo, se encuentra integrada por las áreas de estudio o líneas curriculares, las materias en orden lógico-secuencial, la distribución del tiempo (semestres) y la carga horaria por materia y por área (Díaz et al., 1990).

Las áreas de formación son una forma de organización curricular que articula e integra los conocimientos y experiencias de aprendizaje, su diseño favorece el manejo eficiente de los procesos característicos correspondientes. Cada área representa agrupamientos que toman en cuenta, en diferente grado, criterios pedagógicos, epistemológicos e institucionales. En unos casos posibilitan acercamientos o nexos interdisciplinarios por afinidad de manera explícita, mientras que en otro surge procurando articular procesos comunes que dan curso a experiencias o vivencias valiosas, en torno de metas vinculadas a dimensiones del desarrollo integral de los estudiantes. 


\subsection{Ejes curriculares}

Tanto las áreas disciplinarias como los ejes curriculares, constituyen el primer nivel de selección y organización del contenido, a los que pueden sumarse los ejes temáticos transversales. Una forma práctica de llegar a ellos es mediante el sistema de competencias que funciona sobre la base de que los sujetos educativos pueden demostrar qué hacen (Delgado, 2008:13).

A través de los ejes curriculares se fijan propósitos particulares y se crean sus propias reglas de operación de acuerdo con las características del plan y los programas de estudio, el perfil del personal académico que lo integra y las circunstancias de la unidad formadora a la que esté adscrito. Se sistematizan los criterios para la creación y supresión de ejes y los requisitos de pertenencia y permanencia de los profesores en los mismos, pero se hace hincapié en la necesidad de poner atención a los resultados de los procesos enseñanza-aprendizaje, para fortalecer el desarrollo de la docencia.

\subsection{Ejes transversales}

Los proyectos curriculares de las instituciones educativas contemplan en su diseño la incorporación de áreas o ejes de formación sobre las cuales se direcciona la operatividad del currículo. En armonía con el resto de los componentes curriculares, los ejes transversales presentan un carácter abierto o flexible y como principio didáctico implican para el cuerpo docente un tamiz de lectura y reflexión crítica de objetivos, contenidos, relaciones comunicativas, metodología en todas las actividades, proyectos y demás programaciones pedagógicas (Liendo \& Lúquez de Camacho, 2007:9).

Moreno (2004:6) citado por Velásquez Sarria, 2009:37 entiende la transversalidad curricular como el conjunto de características que distinguen un modelo curricular cuyos contenidos y propósitos de aprendizaje van más allá de los espacios disciplinares y temáticos tradi- cionales, desarrollando nuevos espacios que en ocasiones cruzan el currículo en diferentes direcciones, en otras sirven de ejes a cuyo alrededor giran los demás aprendizajes, o de manera imperceptible y más allá de límites disciplinares impregnan el plan de estudio de valores y actitudes que constituyen la esencia de la formación personal, tanto en lo individual como en lo social.

De tal forma que con ello se incorporan la multidisciplinariedad, interdisciplinariedad y transdisciplinariedad en la estructura del currículo de estudios y en el campo operativo en el abordaje de las diferentes temáticas ambientales a través de los cursos que se proporcionan de acuerdo al diseño curricular. Esto permite evidenciar que la universidad debe encaminar esfuerzos a la inclusión del componente ambiental desde los currículos de estudio, pues la misma se encuentra contemplada dentro del Plan Estratégico ${ }^{2}$, pero no como un eje transversal de formación, lo que ha influido en el hecho de que los avances hasta la fecha se den solamente en algunas unidades académicas que de acuerdo a su naturaleza tienen vinculación con el medio ambiente.

García del Pino citado por Rabino \& Darwich, 2009 señala que: La transversalidad de la educación ambiental, es equivalente a diseñar y organizar los contenidos, procedimientos y objetivos del currículo en torno a ejes referenciales de claro contenido ambiental cuyo valor educativo corresponde con el actual individuo a participar activamente en la construcción de una sociedad en equilibrio con su medio ambiente.

Las diferentes áreas del currículo de estudios deben contribuir al desarrollo de la ambien-

2 El Plan Estratégico USAC-2022, contempla como marco general para su implementación la vinculación de la universidad y sociedad, sus objetivos y acciones están dirigidos a dar respuesta a las necesidades de la sociedad guatemalteca. Por lo que se convierte en un proceso permanente en el marco de la planificación de áreas, ejes y líneas estratégicas que se orientan hacia un escenario futurible a mediano y largo plazo para el año 2022. 
talización curricular. Es por ello que los temas transversales deben:

$\checkmark \quad$ Hacer referencia a cuestiones actuales de gran trascendencia para la vida individual y social, frente a las que conviene generar posiciones personales y colecticas (salud, paz, vida en sociedad, etc.)

$\checkmark$ Contribuir a la educación integral del individuo

$\checkmark \quad$ Integrar las temáticas propias de los distintos temas transversales ya que deben ser complementarias e interdependientes.

$\checkmark$ Impregnar las diferentes áreas del conocimiento

\section{$\checkmark \quad$ Tener un carácter abierto}

La incorporación de estos principios en los ejes del diseño curricular permitirá garantizar la inclusión de la educación ambiental y su operatividad en la implantación del currículo de estudios en la Carrera de Pedagogía.

\subsection{Perfil de ingreso}

El perfil de ingreso orienta la definición de los criterios e instrumentos de selección de los estudiantes a ingresar en la universidad, además define el tipo de alumno que se espera formar en el transcurso de la carrera a nivel técnico. Se considera que a su ingreso a estudios superiores el alumno ha alcanzado las competencias deseables que según el diseño curricular del Ministerio de Educación el alumno ha alcanzado en la educación media, ciclo diversificado y que de acuerdo a los rasgos individuales de cada estudiante son manifiestos en el proceso de admisión a nivel superior.

Para su inscripción en la Carrera de Profesorado de Enseñanza Media en Pedagogía y Técnico en Administración Educativa, se requiere que el estudiante cumpla con los requisitos generales de admisión que se establecen en la universidad: La prueba de orientación voca- cional, conocimientos básicos específicamente del curso de lenguaje y los aspectos administrativos requeridos en control académico del CUNSURORI, así como los requeridos por la Carrera en relación a pruebas de conocimientos específicos donde se realiza una prueba escrita sobre liderazgo y entrevista personal.

Se requiere además que el estudiante posea características deseables respecto a características deseables, capacidades, habilidades y actitudes determinadas en el currículo de estudio de la carrera.

\subsection{Perfil de egreso}

Se considera que el perfil de egreso es la descripción de las características principales que deberán tener los educandos como resultado de haber transitado por un determinado sistema de enseñanza-aprendizaje (Arnaz, 1990). El perfil de egreso se convierte en una descripción del desempeño esperado de un egresado a quien la unidad formadora certifica en términos de objetivos y/o competencias alcanzadas en el proceso formativo, lo que evidencia el compromiso de la institución con el contexto social en donde habrá de desempeñar su labor profesional el estudiante.

Al elaborar el perfil del egresado se debe describir cómo será el profesional producto del sistema para el cual estamos elaborando un currículum, tendremos una caracterización de cómo deberían ser los alumnos egresados y las condiciones expresas que habrán de manifestar para la atención de las necesidades del contexto donde habrán de desempeñarse (Arnaz, 1990). Por lo que conocer el contexto social en donde habrá de desempeñarse el futuro profesional es indispensable en esta tarea, debido a que de esta manera se está en la posibilidad de vincular el perfil de egreso con el contexto de la sociedad en la que ejercerá el futuro profesional, por lo que se debe tomar en cuenta factores sociales, laborales, económicos y políticos en su diseño. 


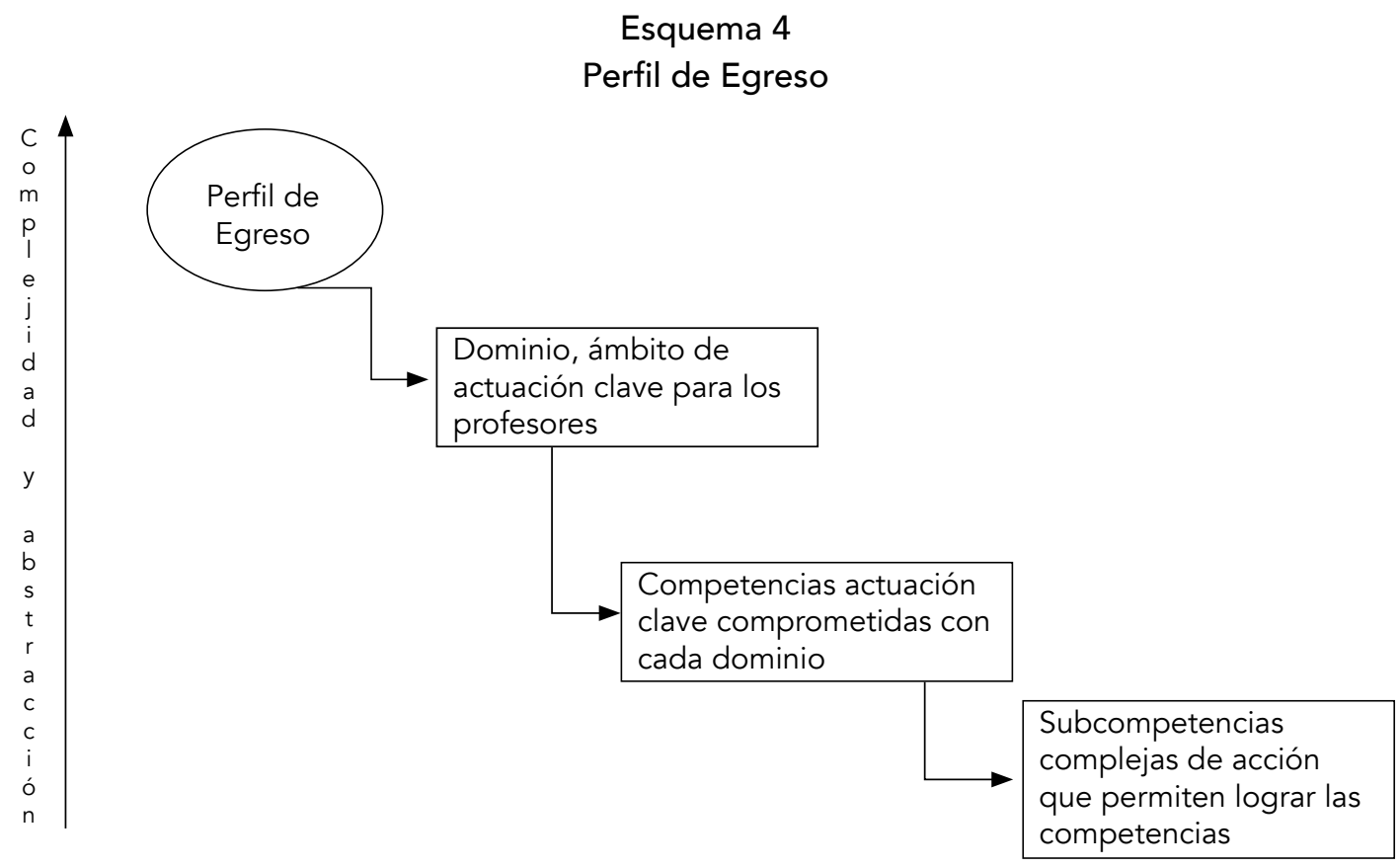

Fuente: Arnaz, 1990.

Los perfiles de egreso se encuentran conformados por los conocimientos y habilidades así como también por las actitudes, elementos que operativamente delimitan el ejercicio profesional. En efecto, los perfiles son una descripción preliminar del egresado que en congruencia con los objetivos curriculares complementen la calidad del profesional que se desea formar.

\subsection{Programas bases de los cursos}

El plan de estudios es una descripción general de lo que será aprendido por el estudiante y cuánto tiempo requiere para ello. Su función es la de informar a profesores, educandos y administradores sobre lo que ha de aprenderse y el orden que seguirá en el proceso. Ela- borar el plan de estudios implica tres actividades: seleccionar los contenidos, derivar de los objetivos generales los objetivos específicos a partir de los cuales se estructurarán los cursos y desglosar los que formarán parte del plan de estudios. (Aguilar \& Vargas, 2011:56).

La elaboración de los programas básicos o de cursos, guías programáticas o cartas descriptivas significa hacerse de guías detalladas para cada parte del plan de estudios, el cual puede estar compuesto de materias, módulos, áreas, etc. De cada una de ellas se necesitará una carta descriptiva que sirva para orientar a profesores y alumnos acerca de qué es lo que estos tendrán que aprender y cómo podrá lograrse tal cosa (Arnaz, 1990:16). 
Esquema 5

Elaboración de las Guías Programáticas

Elaboración de las guías programáticas o cartas descriptivas

Fuente: Arnaz, 1990:18

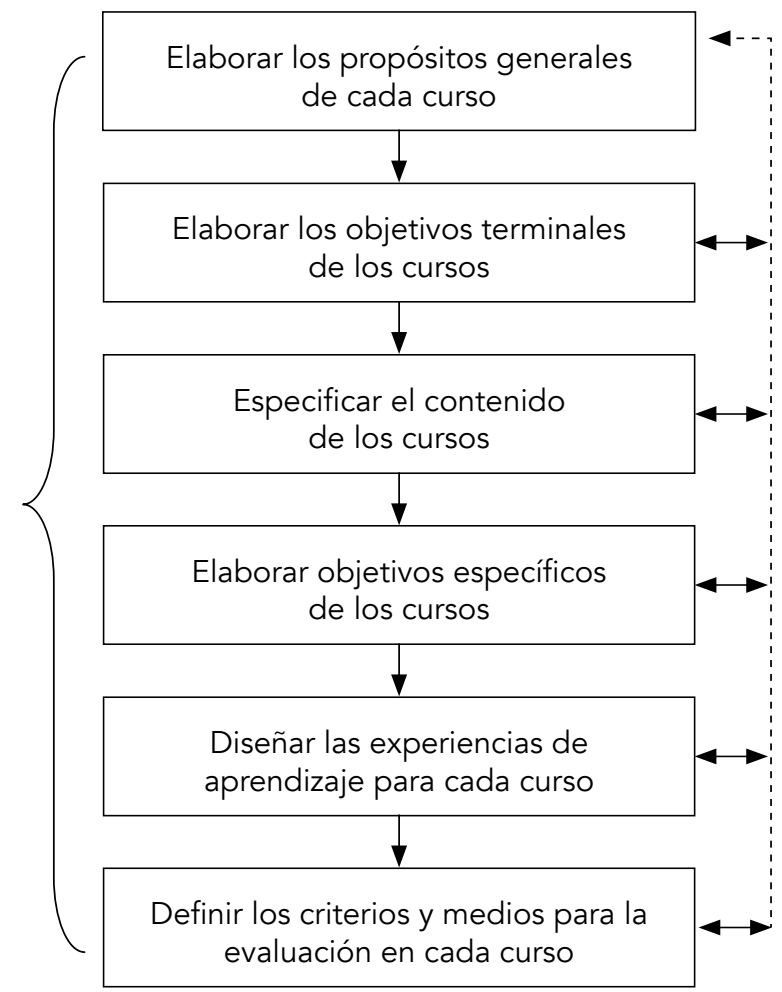

Los cursos son la parte operativa del diseño curricular, por tanto, la estructuración de las guías programáticas de cada uno de ellos debe contener expresamente la temática a desarrollar mediante el establecimiento de objetivos generales y específicos que orienten del diseño y desarrollo de las experiencias de aprendizaje, definiendo los medios adecuados para la implementación de la evaluación en cada asignatura.

\section{Los diseños curriculares en la Universidad de San Carlos de Guatemala y la inclusión de la dimensión ambiental}

La Universidad de San Carlos de Guatemala contempla la promoción del desarrollo humano sostenido y planificación de su desarrollo curricular en función del contexto social y las necesidades que presenta la sociedad guate- malteca. Los diseños curriculares en las unidades formadoras se encuentran construidos en relación a las disposiciones emanadas por la universidad, es así como el Plan Estratégico USAC-2022 en su línea estratégica A.2.2. se refiere al sistema de actualización curricular; aspecto en el que la inclusión de la dimensión ambiental se hace necesaria de acuerdo a los compromisos asumidos por la USAC.

En cuanto a la educación superior, el Seminario Taller "La Universidad y su Compromiso con el Medio Ambiente", desarrollado en 1999 por REDFIA, representa en materia de educación superior una experiencia de colaboración interuniversitaria sobre la temática ambiental y de desarrollo sostenible. El objetivo fue generar mecanismos de coordinación, compromisos y contribuciones en cinco áreas consideradas clave para la educación supe- 
rior y su proyección sobre el uso sostenible y protección del medio ambiente y los recursos naturales:

$\checkmark$ Reforma del diseño curricular para lograr la transversalidad del componente ambiental.

$\checkmark$ Fortalecimiento de los postgrados en medio ambiente y desarrollo sostenible.

$\checkmark$ Fortalecimiento, desarrollo, vinculación y divulgación de la investigación socio-ambiental para crear normas ambientales.

$\checkmark$ Capacitación ambiental comunitaria.

Educación ambiental para el desarrollo sostenible (Ministerio de Ambiente y Recursos Naturales, Ministerio de Educación, 2003).

Abordar el enfoque ambiental planteado en la planificación estratégica de la USAC es un reto que puede asumirse a partir de un proceso de ambientalización curricular, que es una estrategia metodológica, un conjunto de acciones orientadas a incidir en cualquier elemento del currículo con la intención definida de: propiciar la inclusión de conocimiento orientados al logro del desarrollo sustentable con un enfoque integrador y promover valores positivos hacia el medio (solidaridad, responsabilidad, compromiso, respeto, hacia toda forma de vida, convivencia armoniosa, entre otros), (Guier, et.al., 2004) a través de que se incorpore la temática como un eje transversal de formación de las áreas curriculares.

La inclusión de la dimensión ambiental a la educación formal a través del currículo de estudios genera la oportunidad de abordar la problemática ambiental desde la educación escolarizada. La ambientalización curricular es un proceso continuo en el que la producción de conocimiento se encuentra en correspondencia con la formación de profesionales comprometidos en la construcción de un nuevo paradigma hombre/naturaleza, en donde la axiología es un factor indispensable. Está relacionada particularmente con la educación formal, la cual tiene planes de estudio sistematizados que se denomina currículo en las enseñanzas universitaria y básica obligatoria, de manera que la población que es intervenida en el proceso de ambientalización son los alumnos y alumnas del Sistema Educativo Nacional. (Guier, et al. 2004:10).

Los currículos de estudios de los diferentes niveles que integran el Sistema Educativo Nacional (SEN) permiten la inclusión de la temática referida al ambiente de acuerdo a los componentes estructurales del mismo, el impacto de la ambientalización curricular a los pensum de estudios se da en el planeamiento, ejecución y desarrollo del hecho educativo en el salón de clases, aspectos que se evidencian en el currículo escolar sistematizado de la institución educativa. Sin embargo, hay que tomar en consideración el currículo oculto y el modelo de desarrollo imperante en el país que condiciona la formación de valores y la relación socio-económica que el individuo establece con el ambiente.

En el ámbito educativo la ambientalización curricular se establece como una estrategia metodológica, un conjunto de acciones orientadas a incidir en cualquier elemento del currículo con la intención definida de: propiciar la inclusión de conocimientos orientados al logro del desarrollo sustentable con un enfoque integrador. Promover valores positivos hacia el medio (solidaridad, responsabilidad, compromiso, respeto hacia toda forma de vida, convivencia armoniosa, entre otros) (Guier, et.al. 2004).

Por lo que su inclusión dentro del proyecto curricular y/o pensum de estudios debe abordarse de forma holística, para lo cual debe considerarse incorporar contenidos ambientales al currículo de estudios en las diferentes materias que intervienen en la formación del alumnado que permitan la generación de competencias a partir de conceptos, procedimientos y actitudes que impacten al estudiante en su manera de actuar armónicamente con el medio ambiente. 
Ambientalizar el currículum no significa la creación de una nueva asignatura, ni siquiera la utilización del entorno escolar como un recurso educativo para el desarrollo de distintas materias o temas. Supone por el contrario, tomar los principios ambientales como los educativos. Esto implica tenerlos en cuenta en todas las decisiones que se tomen en nuestra práctica docente. (Rabino \& Darwich, 2009).

La ambientalización curricular permite por tanto la inclusión de ejes transversales al currículo de estudios a través de los cuales permee la educación ambiental en las diferentes asignaturas del pensum de estudios de la unidad académica, con lo cual se pretende generar procesos de formación a fin de que los estudiantes posteriormente trasladen a la actividad docente los principios, valores, competencias que en relación a la temática ambiental han asimilado en su formación académica.

La inclusión de la ambientalización curricular puede incorporarse al currículo a través del diseño de competencias. Se considera que el término competencia se refiere a una combinación de destrezas, conocimientos, aptitudes y actitudes, y a la inclusión de la disposición para aprender, además del saber cómo (Comisión Europea, 2004), es un término complejo que implica la adquisición dominio de conocimientos cuyo aprendizaje tiende a su utilización de manera integrada y articulada en actividades del diario vivir. Se pretende que las competencias alcanzadas por el estudiante en diferentes áreas del conocimiento, le posibiliten para saber utilizarlas y activarlas con el fin de responder a diferentes contextos y momentos de su vida cotidiana.

Los diseños curriculares planteados por competencias, hacen referencia a la elaboración de planes de estudio que de acuerdo a su nivel se orienten a facilitar en los alumnos el desarrollo de las competencias, según los contenidos programáticos, contemplados en el pensum de estudios, se debe considerar para ello las necesidades del medio social, de las unidades empleadoras y dentro de la carga académica que la universidad establece. El Informe Delors auspiciado por la UNESCO plantea los cuatro pilares de la educación por competencias:

$\checkmark \quad$ Aprender a conocer: Es la capacidad que tiene para la comunicación la comprensión y adquirir conocimiento, estimulando la creación de un sentido crítico con autonomía. Se requiere un aprendizaje para toda la vida.

$\checkmark$ Aprender a hacer: El aprendizaje como práctica, vinculado al futuro del mercado de trabajo. Conocimientos como activos.

$\checkmark \quad$ Aprender a vivir juntos: Descubrirse y aceptar las diferencias. Trabajo colaborativo.

$\checkmark$ Aprender a ser: Generar la responsabilidad individual, dando oportunidades a partir de lo que se es (Delors, 1997).

El currículo centrado en competencias puede asumir diferentes enfoques, de acuerdo a la priorización de los aspectos referidos en su estructuración. Encontramos por tanto un enfoque transversal en donde se pretende promover aprendizajes dinámicos en los cuales se persigue la formación de competencias para la vida o en la interdisciplinariedad de conocimientos.

El enfoque curricular pretende una perspectiva integral de la enseñanza conjugando conocimientos, destrezas, actitudes y valores que permitan la formación del estudiante, a través del aprendizaje significativo que lo posibilite para enfrentar las diversas situaciones de la vida social a nivel individual y grupal en las cuales pueda aplicar lo aprendido en el salón de clases y otras actividades curriculares.

Por lo que el diseño curricular basado en competencias no puede, ni debe limitarse a los saberes, debe articularse con las necesidades del contexto en donde se desarrolla el hecho educativo y las tareas adecuadas para su utili- 
zación. El papel integrador de las competencias permite la organización de los contenidos en función de lo que se espera que el estudiante sea capaz de adquirir y hacer.

El Diseño Curricular que requiere el CSUCA para la acreditación de las carreras que se atienden en la USAC es un "Enfoque por Competencias", lo que supone que los egresados habrán de desempeñarse de forma eficiente en la realización de trabajo docente, debido a que el perfil de egreso de los mismos le permitió la adquisición de conocimientos, habilidades, destrezas y comportamientos que son observables en su práctica profesional y que contribuirán al alcance de objetivos y metas de la institución educativa en donde labora.

La competencia por tanto se convierte en una capacidad laboral, medible, necesaria para realizar un trabajo eficazmente a través de la producción de resultados deseables en la institución en la cual se labora y que repercutirán en el alcance de metas y objetivos de la organización que se vinculan estrechamente con la eficacia del rendimiento en el trabajo.

\section{Propuesta de Diseño Curricular} para la Carrera de Profesorado de Enseñanza Media en Pedagogía y Técnico en Administración Educativa del Centro Universitario de Sur Oriente CUNSURORI-Jalapa

La Carrera de Profesorado de Enseñanza Media en Pedagogía y Técnico en Administración Educativa del Centro Universitario de Sur Oriente CUNSURORI-Jalapa, nace en Jutiapa el 2 de enero del 2007, su apertura obedece a las disposiciones emanadas del Consejo Superior Universitario contempladas en el acta 09-2002 de fecha 20 de abril de 2002 punto octavo, donde se acordó el traslado de las secciones departamentales a los centros regionales universitarios. Sin embargo en el departamento de Jutiapa no se contaba con Centro Universitario, por lo que la cobertu- ra fue proporcionada por el CUNSURORI en Jalapa.

Es preciso mencionar que fue la única sección departamental que acató la disposición del Consejo Superior y que gracias a las gestiones del licenciado Luis Alfonso de Paz Morales en establecer los contactos con el CUNSURORI empieza a funcionar la carrera en Jutiapa

En sus acuerdos expone: $1^{\circ}$. Aprobar la propuesta de integración de las Secciones Departamentales de la Facultad de Humanidades a los Centros Regionales, incorporando dichas secciones a la estructura administrativa y académica de los mismos sin cambiar la ubicación física de las sedes municipales actuales. $2^{\circ}$. Dicha integración debe hacerse en forma gradual y con fundamentos de orden académico, administrativo y financiero, acorde con las políticas universitarias de descentralización y considerando las recomendaciones de la Comisión nombrada por el Consejo Superior Universitario para la implementación de esta propuesta, para lo cual fue designada la Dirección General de Docencia y a la Dirección General Financiera ejecutar, con el apoyo de las demás dependencias que fueran pertinentes la operativización de este proceso, el cual fue iniciado en el primer año lectivo del ciclo académico 2003, y se consideró que se debía avanzar en forma gradual hasta completar la integración total.

Los hallazgos de la comisión se retoman en sesión del Consejo Superior Universitario celebrada el 4 de octubre de 2006 en donde se contempla en el inciso 6.1 el traslado de las secciones departamentales a los Centros Regionales a partir de enero de 2007. Dicho punto de acta es retomado por el Consejo Directivo del CUNSURORI y en el Acta No. 24-2008 punto cuarto acuerda: Avalar la apertura de las Carreras de Profesorado de Segunda Enseñanza en Pedagogía y Administración Educativa y Profesorado de Segunda Enseñanza en Pedagogía e Investigación Educativa, de la Facultad de Humanidades, a partir del 
2009, plan fin de semana, en el CUNSURORI, según acuerdo en mención.

El 7 de febrero de 2009 tuvo lugar la inauguración de la Carrera con una matrícula estudiantil de 250 estudiantes distribuidos en cuatro secciones bajo la atención de 8 docentes y la Coordinación de Carrera por la licenciada Mirna Orellana Cardona, plan fin de semana con horario de funcionamiento de 7:30 a 17:30 horas y la apertura escalonada de los semestres hasta completar la carrera.

El pensum de estudios implementado corresponde a la adaptación del pensum de la sede central y de la sección de Jutiapa, por lo que se inicia el proceso formativo de los estudiantes de forma escalonada, con la apertura de los semestres conforme el avance de la primera promoción. A partir del segundo año las cuatro secciones fueron reducidas a dos y desde entonces a la fecha se trabaja en la sede Jalapa con dos secciones por semestre.

En el 2010 el Director del CUNSURORI establece contactos con autoridades municipales para la apertura de la Carrera al interior del departamento, con lo que se obtiene el aval del alcalde, doctor Hugo Loyd, y su corporación municipal y se establece un compromiso en la creación de la sede de la Carrera de Pedagogía en Mataquescuintla. El primer año de funcionamiento (2010) se trabaja con el presupuesto de la Municipalidad de Mataquescuintla y en el 2012 se absorbe el presupuesto por la universidad a excepción de los servicios secretariales que a la fecha son cubiertos por dicha dependencia.

La sede de Mataquescuintla inicia funciones en febrero del 2010 en las instalaciones de la Escuela Urbana Hugo Loyd, a la fecha cubre hasta el décimo semestre de la carrera y se espera que para 2015 estén abiertos todos los semestres que integran el pensum de estudios.

La sede de San Pedro Pinula es de reciente apertura (2013); empezó a funcionar con los gastos de nómina docente cubiertos por la Municipalidad del lugar. A inicios del 2014 la Universidad absorbe el presupuesto de pago docente y la Municipalidad paga los servicios secretariales; actualmente atiende a una población de 20 estudiantes. Se han realizado acciones encaminadas a incrementar la matrícula estudiantil para el 2015.

En cuanto a diseño curricular funciona con la pensa de estudios de la Facultad de Humanidades y la sede de Jutiapa, pero no se cuenta con un currículo de estudios que oriente dicho proceso, por lo cual la presente propuesta pretende ser un instrumento de apoyo en la construcción de un currículo de estudios que recolecte el funcionamiento actual de la carrera.

La estructuración de un diseño curricular implica la expresión clara y precisa de cada uno de los aspectos vinculados a: pensa de estudios, contenidos, áreas y ejes de formación, procesos de enseñanza y aprendizaje y el establecimiento de normas básicas de especificación sobre: procesos de evaluación y mejoramiento de los contenidos y procesos de enseñanza y aprendizaje. Se constituye en un instrumento común en la interacción de los profesionales que forman el equipo docente de la carrera donde laboran.

Se considera pertinente la estructuración del Diseño Curricular de la Carrera de Profesorado de Enseñanza Media y Técnico en Administración Educativa del Centro Universitario de Sur Oriente CUNSURORI-Jalapa, para lo cual se hace una propuesta en donde se incorpora la ambientalización curricular a través de la inclusión de la dimensión ambiental al currículo.

Se plantea la construcción de un modelo por competencias, en el ámbito de la formación universitaria, la idea de competencia posee un carácter integrador. Su definición surge de las características de un determinado perfil profesional, del análisis pormenorizado de una actividad o puesto de trabajo, de las demandas específicas que se hacen a los proveedores de 
formación (las nuevas funciones que se pretenden cubrir con esa titulación) y, finalmente, de los propósitos formativos que se pretenda dar a una titulación o plan curricular (Pavié, 2011).

Entendida la competencia en sus distintos niveles: saber (datos, conceptos conocimientos), saber hacer (habilidades, destrezas, métodos de actuación), saber ser (actitudes y valores que guían el comportamiento) y saber estar (capacidades relacionadas a la comunicación interpersonal y el trabajo cooperativo), con lo cual se estaría dotando al estudiante de las capacidades necesarias para su desempeño en la complejidad del contexto social. Para lo cual se toma en cuenta la integración de la competencia en la incorporación de conocimientos, habilidades, destrezas, actitudes y valores al proceso formativo del estudiante.

Además, se enfatiza en el enfoque holístico dentro del diseño curricular a través del cual el estudiante adquiera los atributos necesarios que en base al modelo por competencias se articule en el proceso de enseñanza-aprendizaje, en las cuatro áreas diseñadas en las que se ubican los cursos del pensum de estudios. Estas son: area de investigación, área de realidad nacional, área administrativa y área pedagógica, en las cuales se encuentran los ejes transversales de educación y educación ambiental cruzando el pensum de estudios e impregnando la formación del estudiante.

Se presenta la construcción de los perfiles de ingreso y de egreso en congruencia con los objetivos de formación planteados para la carrera, con lo que los cursos del pensum de estudios y los créditos de cada uno responden al diseño propuesto.

Cabe señalar, que la propuesta se enmarca en los lineamientos técnicos y legales que contempla la Universidad de San Carlos de Guatemala y pretende ser un aporte para su implantación dentro de la carrera. 


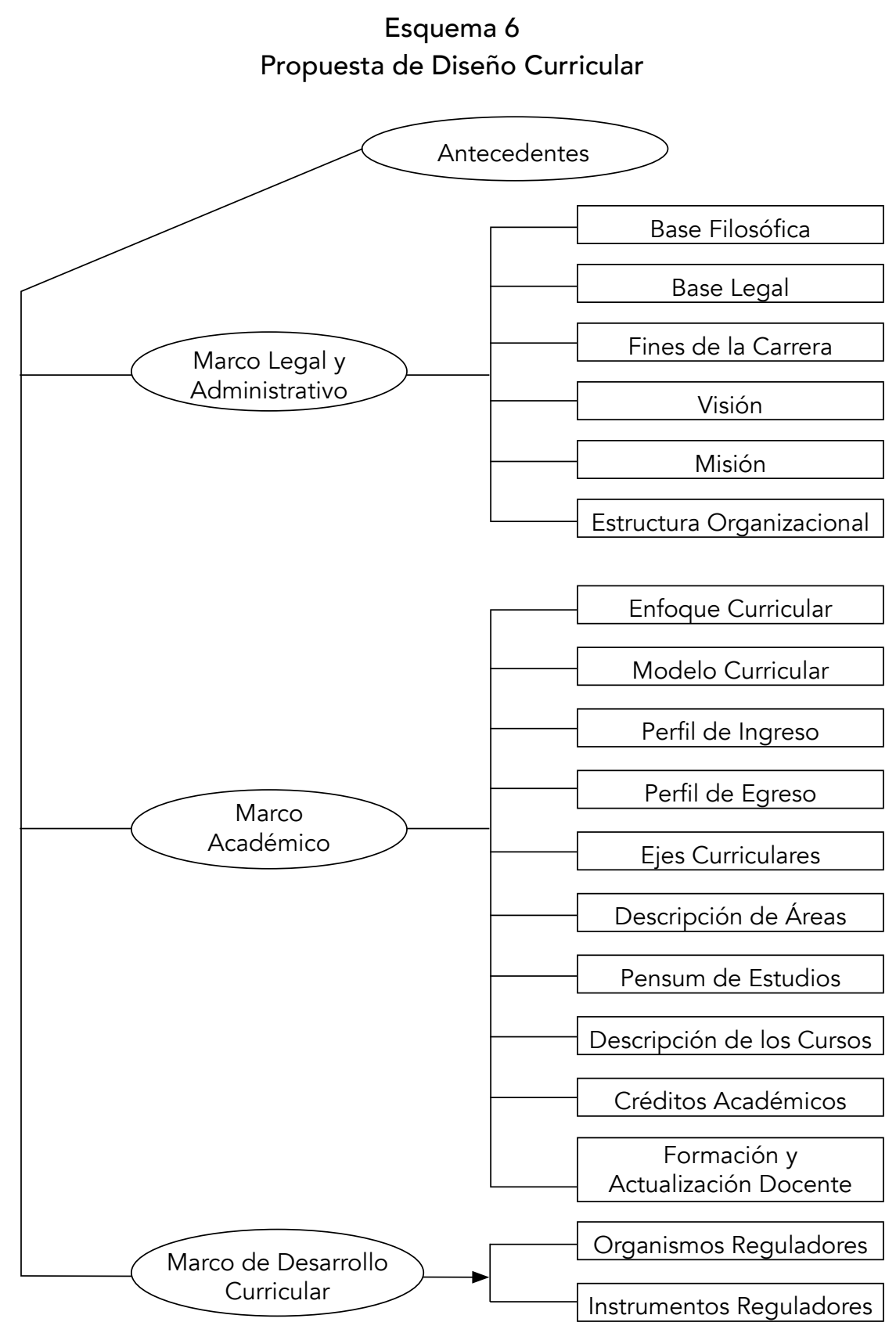

Fuente: Elaboración propia, febrero, 2015.

Los elementos generales considerados para el diseño curricular dentro de la USAC, se encuentran conformados por: Antecedentes, marco legal y administrativo, marco académico y marco de desarrollo curricular, mismos que se deben contemplar para la estructuración de diseños curriculares y/o readecuación curricular y que en el caso particular de la Carrera de Pedagogía a nivel de pre-grado se abordan en el documento de propuesta de diseño curricular.

Destaca el enfoque curricular holístico, que es el que se adopta dentro del diseño curricular, el cual está estructurado y organizado por cinco componentes: dimensión, escenario, reglas, control y cultura, cada uno contempla dentro de sí elementos internos y externos. 
$\checkmark \quad$ Dimensión: Se considera en este aspecto la dimensión pedagógica constituida por tres elementos: uno interno denominado académico, otro externo llamado social y un tercer elemento que emerge de la interrelación social-académico (externo-interno).

$\checkmark \quad$ Escenario: El componente escenario está estructurado y organizado por tres elementos: uno interno denominado aula, otro externo llamado sociedad, y el tercer elemento que emerge de la interrelación aula sociedad (interno-externo) conocido como universidad (latente).

$\checkmark$ Reglas: El componente reglas, estructurado y organizado por los elementos: regla potencial (interno), y regla pensada o admitida (latente) que emerge de la interrelación de las otras dos reglas potencial-real (interno-externo).

$\checkmark \quad$ Control: El componente control está constituido por tres elementos: uno denominado liderazgo (externo), otro llamado desarrollo humano (interno) y el tercer elemento emerge de la interrelación desarrollo humano-liderazgo (interno-externo) denominado administración (latente).

$\checkmark$ Cultura: El componente cultura está estructurado por tres elementos: uno denominado problema (externo), otro denominado contenido (interno) y el tercero emerge de la interrelación problema-contenido (externo-interno) denominado objeto (latente).

En el enfoque holístico o integrado, competente es la persona que posee atributos necesarios (conocimientos, habilidades, actitudes y valores), para el desempeño del trabajo de acuerdo con la norma apropiada. Basada en una visión que trata de integrar lo mejor de los procesos anteriores. Conecta el enfoque de los atributos generales con el contexto en el que éstos se ponen en juego (Pavié, 2011).
Con este modelo se pretende la formación integral del estudiante dentro del contexto socioeconómico y cultural en el cual se desarrolla el hecho educativo, de tal forma que el egresado adquiera las competencias necesarias para ejercer la profesión docente de acuerdo a las exigencias del mercado laboral y la situación educativa del país.

Respecto al mercado laboral en donde habrá de emplearse el profesional egresado de la Carrera de Pedagogía, la propuesta curricular se enmarca en la formación de Profesores de Enseñanza Media cuyo perfil se vincula a cumplir los requerimientos que el MINEDUC contempla actualmente en el contexto nacional y que derivan del proceso de Reforma Educativa y Transformación Curricular. Al respecto, se enfatiza en la formación técnica, científica y humanista de los estudiantes, lo que les permita la adquisición de competencias, herramientas y habilidades que les ayuden a implementar en el aula el nuevo paradigma educativo con el cual se trabaja en el nivel medio a través del Currículo Nacional Base, CNB.

\section{Conclusiones}

El diseño curricular dentro de una institución educativa materializado en un documento escrito es indispensable pues incorpora aspectos vinculantes entre el proceso formativo del estudiante a las bases legales y filosóficas de la universidad, con lo cual el diseño de los perfiles de egreso, misión y visión de la carrera se encontrarán en correspondencia con el alcance de la institución a nivel nacional.

El sector docente debe conocer los elementos filosóficos y académicos de la unidad formadora para la planificación y construcción de saberes articulados a los requerimientos de la formación profesional que egresa de la carrera y el planeamiento educativo en los diferentes niveles de concreción que contribuyan a su alcance.

La incorporación de la dimensión ambiental al currículo de estudios de la Carrera de Pe- 
dagogía es viable si se considera su sustento en el marco legal y administrativo de la USAC, específicamente lo contenido en el Marco Filosófico, Marco de Realidad y Necesidades de la Sociedad guatemalteca, Marco Académico, Visión, Misión y el Plan Estratégico de la Universidad de San Carlos de Guatemala, USAC.

\section{Referencias bibliográficas}

Ader, E. E., 1993. La planificación educativa: Conceptos, métodos, estrategias y técnicas para los educadores. Argentina: Magisterio del Río de la Plata.

Aguilar Morales, J. E. \& Vargas Mendoza, J. E., 2011. Planeación educativa y diseño curricular: Un ejercicio de sistematización. Boletín Electrónico de Investigación de la Asociación Oaxaqueña de Psicología A.C., 7(1), pp. 53-64.

Arnaz, J., 1996. La planeación curricular. Segunda edición ed. México: Trillas.

Comisión Europea, 2004. Competencias clase para un aprendizaje a lo largo de la vida: Un marco de referencia europeo. [En línea] Available at: http://www.educastur.princast.es/info/calidad/indicadores/doc/comisión euro pea.pdf. [Último acceso: 10 febrero 2014].

Delgado M., E., 2008. Los contenidos programáticos y la formación integral en el diseño curricular. Posgrado y Sociedad, 8(1), pp. 89-121.

Delors, J., 1996. La educación encierra un tesoro: Informe a la UNESCO de la comisión internacional sobre la educación para el siglo xxi. [En línea] Available at: http://www.educa.madrid. org/cms tools/files/6bebccef-888c-4dd6b8c1-d0f617656af/La educacion.pdf

Díaz Barriga, A., Lule, M., Rojas, S. \& Saad, S., 1990. Metodología del diseño curricular para educación superior, México: Trillas.
González de Ochaeta, B. E., 2005. Las caracteristicas de la estructura curricular y programas de la Universidad de San Carlos de Guatemala. Guatemala: USAC/IESALC.

Guier, E., 2000. Educación Ambiental: Fundamentos, síntesis histórica en Costa Rica y algunos elementos para la integración del currículo. Costa Rica: Programa Educación Ambiental, UNED.

Liendo Durán, Z. \& Lúquez de Camacho, P., 2007. Eje transversal valores: Epistemología y fundamentos curriculares en la práctica pedagógica de educación básica. Laurus, 13(25), pp. 82-113.

Mauri, T., 1990. Curriculum y enseñanza en el curriculum del centro educativo. Barcelona: ICE/Horsori.

Meza Morales, J. L., 2012. Diseño y desarrollo curricular. México: Red Tercer Milenio.

Ministerio de Ambiente y Recursos Naturales, Ministerio de Educación, 2003. Política Nacional de Educación Ambiental. Guatemala: s.e..

Pavié, A., 2011. Formación docente: Hacia una definición del concepto de competencia profesional docente. Electrónica Interuniversitaria de Formación del Profesorado, 14(1), pp. 67 - 80.

Posner, G., 1998. Análisis de curriculum. Segunda edición ed. Santa Fe de Bogotá, Colombia: McGraw-Hill, Interamericana S.A..

Rabino, M. \& Darwich, M. P., 2009. Ambientalizar el curriculum: Una propuesta para mejorar la calidad de vida. Argentina, Departamento de Ciencias Exactas y Naturales FaHCE (UNLP), pp. 317-324.

Rohlehr, B. A., 2006. Características del currículo y la gestión curricular: Un estudio, Santiago, Chile: OREAL/UNESCO. 
